\title{
Generation of squeezed states of microwave radiation in a superconducting resonant circuit
}

\author{
A.M. Zagoskin ${ }^{1,2,3}$, E. Il'ichev ${ }^{4}$, M. W. McCutcheon ${ }^{5}$, Jeff Young ${ }^{2}$, and Franco Nori ${ }^{3,6}$ \\ (1) Department of Physics, Loughborough University, \\ Loughborough, Leics LE11 3TU, UK \\ (2) Department of Physics and Astronomy, \\ The University of British Columbia, \\ Vancouver, BC, V6T 1Z1, Canada \\ (3) Digital Materials Laboratory, Frontier Research System, \\ RIKEN, Wako-shi, Saitama 351-0198, Japan \\ (4) Institute of Photonic Technology, \\ P.O. Box 100239, D-07702 Jena, Germany \\ (5) School of Engineering and Applied Sciences, \\ Harvard University, Cambridge, MA 02138, USA and \\ (6) Center for Theoretical Physics, Physics Department, \\ Center for the Study of Complex Systems, \\ The University of Michigan, Ann Arbor, MI 48109-1040, USA
}

\begin{abstract}
High-quality superconducting oscillators have been successfully used for quantum control and readout devices in conjunction with superconducting qubits. Also, it is well known that squeezed states can improve the accuracy of measurements to subquantum, or at least subthermal, levels. Here we show theoretically how to produce squeezed states of microwave radiation in a superconducting oscillator with tunable parameters. The circuit impedance, and thus the resonance frequency, can be changed by controlling the state of an RF SQUID inductively coupled to the oscillator. By repeatedly shifting the resonance frequency between any two values, it is possible to produce squeezed and subthermal states of the electromagnetic field in the (0.1-10) GHz range, even when the relative frequency change is small. We propose experimental protocols for the verification of squeezed state generation, and for their use to improve the readout fidelity when such oscillators serve as quantum transducers.
\end{abstract}


The problem of quantum measurements has recently attracted renewed attention. In quantum mechanics, the extraction of information from a quantum system produces an unavoidable disturbance on it. If the object is initially in an eigenstate of the measured observable, a quantum non-demolition (QND) measurement can be realized, where this disturbance is minimal [1].

One known type of detector for QND measurements is the so-called parametric transducer [1]. A key element of a parametric transducer is an optical or radio-frequency autooscillator. A transducer coupled to a quantum system of interest is designed such that the behaviour of the quantum system changes the phase and/or amplitude of its oscillations. The phase (amplitude) shift provides information about the quantum system's dynamics. With the recent development of superconducting qubits [2, 3], this approach was successfully applied to their study. In particular, transducers (high-quality superconducting tank circuits) were used to measure the state of superconducting flux qubits [4, 5].

It is known that the noise of detectors can be decreased, even below the standard quantum noise level, by employing squeezed states [6, 7, 8]. In this paper we show that a superconducting parametric transducer allows a natural application of this approach, since it can be used both to produce squeezed states and to use them in order to minimize quantum fluctuations. An immediate result of this method's application would be a way to suppress the effective noise temperature of the next-stage amplifier, at least to the nominal temperature of the cooling chamber. We emphasize that existing experimental techniques should be sufficient for the realization of our proposal.

Squeezed states [6, 7, 8] are quantum states in which the dispersion of one variable can be below the standard quantum limit (SQL). More specifically, if a system is described by a pair of dimensionless conjugate variables, $Q$ and $P$, it is in a squeezed state if for some times

$$
\left\langle\Delta Q^{2}\right\rangle \equiv\left\langle Q^{2}\right\rangle-\langle Q\rangle^{2}<1 / 2
$$

The uncertainty principle requires that $\left\langle\Delta P^{2}\right\rangle\left\langle\Delta Q^{2}\right\rangle \geq 1 / 4$, so when one variable is squeezed, the dispersion of the conjugate variable increases. Squeezed states were introduced in the context of quantum optics (see, e.g., Refs. 6, 7, 8]), but were since investigated in a number of other systems, including polaritons [9, 10], phonons [11, 12], Josephson junctions [13] and molecular oscillations [14]. Squeezed states have been successfully generated in 
a Josephson parametric amplifier [15]. A special interest in these states is due to their usefulness in obtaining SQL resolution in imaging and measurement (see, e.g., [6, 7]). Their classical analog can be used to obtain subthermal resolution in mechanical measurements [16].

As described in, e.g., Ref. [17], if a harmonic oscillator is in a coherent state (i.e., a state with equal and minimal uncertainties: $\left.\left\langle\Delta P^{2}\right\rangle=\left\langle\Delta Q^{2}\right\rangle=1 / 2\right)$, a sudden change of the oscillator frequency would create a squeezed state (while an adiabatic change would not). The degree of squeezing is given by the ratio

$$
\lambda=\omega / \omega_{0}
$$

of the oscillator frequencies before and after the shift, or its inverse, whichever is less than one. This topic was further investigated in some detail in Refs. [18, 19, 20, 21, 22]. In particular, it was shown [19] that, by repeated and properly timed oscillator frequency shifts, one could reach an arbitrary degree of squeezing, even for $\lambda$ close to unity (neglecting damping, and assuming the frequency shifts to be instantaneous). A general analysis of this situation, under less restrictive assumptions, was given in Ref. [22].

In this paper we consider the possibility of using repeated frequency shifts to produce $\mathrm{GHz}$ squeezed states in a superconducting resonant tank circuit, and the use of these states to improve the sensitivity of such a circuit when employed as a parametric transducer. The parameters of the circuit can be tuned by controlling the state of an RF SQUID inductively coupled to the superconducting oscillator (resonant tank circuit) [4, 5]. This distinguishes our proposal from Ref. [23], where the generation of squeezed states in an RF SQUID with a tunable junction was proposed through a single-step change of the parameters of the junction.

The assumption of an instantaneous switching of the oscillator frequency is convenient for a proof-of-principle analysis, but not sufficient for the discussion of an experimental realization of the effect. Therefore, we start from the density matrix $\rho$ of the tank circuit coupled to the superconducting oscillator, which satisfies the equation

$$
i \partial_{t} \rho=[H(t), \rho]
$$

where $H(t)$ is the Hamiltonian. Additional Lindblad terms in the r.h.s. can be added to account for dephasing and relaxation [8]. 
Initially, $H(0)=\left(\omega_{0} / 2\right)\left(a_{0}^{\dagger} a_{0}+a_{0} a_{0}^{\dagger}\right)$. A change of the oscillator frequency, $\omega_{0} \rightarrow \omega$, transforms the creation/annihilation operators to [17]

$$
a=\frac{\omega+\omega_{0}}{2 \sqrt{\omega \omega_{0}}} a_{0}+\frac{\omega-\omega_{0}}{2 \sqrt{\omega \omega_{0}}} a_{0}^{\dagger} ; \quad a^{\dagger}=\frac{\omega+\omega_{0}}{2 \sqrt{\omega \omega_{0}}} a_{0}^{\dagger}+\frac{\omega-\omega_{0}}{2 \sqrt{\omega \omega_{0}}} a_{0} .
$$

These formulas follow from the requirement that the momentum and position operators do not change. This Bogoliubov transformation can be rewritten [17] as $a_{0} \rightarrow a=U a_{0} U^{\dagger}$, where

$$
U=\exp \left[(1 / 4) \ln \left(\omega / \omega_{0}\right)\left(a_{0}^{\dagger 2}-a_{0}^{2}\right)\right]
$$

The transformation of the Hamiltonian under the unitary transformation (4) is given by

$$
H=U H_{0} U^{\dagger}-i U \partial_{t} U^{\dagger}
$$

here $H_{0} \equiv H(0)$ is the initial Hamiltonian; the last term is the most important in the case of fast frequency changes (see, e.g., [24]). The resulting time-dependent Hamiltonian, expressed in terms of the original creation/annihilation operators, is

$$
H(t)=H_{0}+\frac{\omega(t)^{2}-\omega_{0}^{2}}{4 \omega(t)}\left(a_{0}^{\dagger} a_{0}+a_{0} a_{0}^{\dagger}+a_{0}^{\dagger 2}+a_{0}^{2}\right)-i \frac{\dot{\omega}(t)}{\omega(t)}\left(a_{0}^{\dagger 2}-a_{0}^{2}\right) .
$$

Finally, by moving to the interaction representation with respect to $H_{0}$, we find the Hamiltonian (which, for the sake of briefness, is also denoted by $H(t)$ )

$H(t)=\frac{\omega(t)^{2}-\omega_{0}^{2}}{4 \omega(t)}\left(a_{0}^{\dagger} a_{0}+a_{0} a_{0}^{\dagger}\right)+\frac{\omega(t)^{2}-\omega_{0}^{2}}{4 \omega(t)}\left(a_{0}^{\dagger 2} e^{2 i \omega_{0} t}+a_{0}^{2} e^{-2 i \omega_{0} t}\right)-i \frac{\dot{\omega}(t)}{\omega(t)}\left(a_{0}^{\dagger 2} e^{2 i \omega_{0} t}-a_{0}^{2} e^{-2 i \omega_{0} t}\right)$.

Hereafter, it is convenient to use the coherent state representation [7, 8]. A coherent state $|\alpha\rangle$ is the eigenvector of the annihilation operator with the (complex) eigenvalue $\alpha$ : $|\alpha\rangle: a_{0}|\alpha\rangle=\alpha|\alpha\rangle$, while $\langle\alpha| a_{0}^{\dagger}=\langle\alpha| \alpha^{*}$. Each coherent state is a superposition of an infinite number of Fock states (states with a definite number of photons) and in the classical limit becomes a classical state with definite, time-dependent position and momentum (or other appropriate pair of canonically-conjugate classical variables).

A density matrix $\rho$ can be represented by the Wigner function $W\left(\alpha, \alpha^{*}\right)$. Examples are a coherent state: $\rho_{\zeta}=|\zeta\rangle\langle\zeta| \leftrightarrow W_{\zeta}\left(\alpha, \alpha^{*}\right)=\frac{2}{\pi} e^{-2|\alpha-\zeta|^{2}}$; a thermal state: $W_{T}\left(\alpha, \alpha^{*}\right)=$ $\frac{2}{\pi} \tanh \left(\frac{\omega_{0}}{2 T}\right) \exp \left[-2|\alpha|^{2} \tanh \left(\frac{\omega_{0}}{2 T}\right)\right] ;$ and a number state: $\rho_{n}=|n\rangle\langle n| \leftrightarrow W_{n}\left(\alpha, \alpha^{*}\right)=$ $\frac{2(-1)^{n}}{\pi} e^{-2|\alpha|^{2}} \mathrm{~L}_{n}\left(4|\alpha|^{2}\right)$. Here $\mathrm{L}_{n}(z)$ is the Laguerre polynomial. The complex variables $\alpha$ and 
$\alpha^{*}$ can be expressed through their real quadrature components, $x, y: \alpha=x+i y, \alpha^{*}=x-i y$, which can be related to directly observable properties of an oscillator (e.g., current and voltage). For our purposes, the usefulness of this representation is due to the fact that there exists a one-to-one correspondence [8] between the action of creation/annihilation operators on $\rho$ and differential operations on $W\left(\alpha, \alpha^{*}\right)$ :

$$
\begin{aligned}
& a_{0} \rho \leftrightarrow\left[\alpha+(1 / 2) \partial_{\alpha^{*}}\right] W\left(\alpha, \alpha^{*}\right) ; \rho a_{0} \leftrightarrow\left[\alpha-(1 / 2) \partial_{\alpha^{*}}\right] W\left(\alpha, \alpha^{*}\right) ; \\
& a_{0}^{\dagger} \rho \leftrightarrow\left[\alpha^{*}-(1 / 2) \partial_{\alpha}\right] W\left(\alpha, \alpha^{*}\right) ; \rho a_{0}^{\dagger} \leftrightarrow\left[\alpha^{*}+(1 / 2) \partial_{\alpha}\right] W\left(\alpha, \alpha^{*}\right) .
\end{aligned}
$$

Therefore the Liouville equation for the density matrix operator is now replaced by a partial differential equation for a c-number Wigner function

$$
i \partial_{t} W\left(\alpha, \alpha^{*}\right)=2 \beta(t)\left[\alpha^{*} \partial_{\alpha^{*}}-\alpha \partial_{\alpha}\right] W\left(\alpha, \alpha^{*}\right)+2\left[\gamma(t)^{*} \alpha \partial_{\alpha^{*}}-\gamma(t) \alpha^{*} \partial_{\alpha}\right] W\left(\alpha, \alpha^{*}\right)
$$

Here we have introduced

$$
\begin{array}{r}
\beta(t)=\frac{\omega_{0}}{4}\left[\left(\frac{\omega(t)}{\omega_{0}}\right)^{2}-1\right] \equiv \frac{\omega_{0}}{4}\left[\lambda(t)^{2}-1\right] ; \\
\gamma(t)=\left[\beta(t)+i \frac{\dot{\omega}(t)}{\omega(t)}\right] e^{2 i \omega_{0} t} \equiv\left[\beta(t)+i \frac{\dot{\lambda}(t)}{\lambda(t)}\right] e^{2 i \omega_{0} t} .
\end{array}
$$

In the presence of dissipation, Eq. (8) will also contain second-order terms, and would be only tractable numerically. Neglecting these terms leads to an immediate simplification, since Eq. (8) is a differential equation of first order and can be solved by the method of characteristics. The characteristics $x(t), y(t)$ satisfy the equations:

$$
\begin{gathered}
\frac{1}{2} \dot{x}=[\operatorname{Im} \gamma(t)] x(t)+\{\beta(t)-[\operatorname{Re} \gamma(t)]\} y(t) \\
\frac{1}{2} \dot{y}=-\{\beta(t)+[\operatorname{Re} \gamma(t)]\} x(t)-[\operatorname{Im} \gamma(t)] y(t) .
\end{gathered}
$$

After finding the solutions $x\left(x_{0}, y_{0}, t\right) ; y\left(x_{0}, y_{0}, t\right)$ (where $x_{0}, y_{0}$ are the initial conditions) and inverting them to obtain $x_{0}(x, y, t) ; y_{0}(x, y, t)$, one obtains the Wigner function for an arbitrary point and time from its value at $t=0$ via

$$
W(x+i y, x-i y, t)=W\left(x_{0}(x, y, t)+i y_{0}(x, y, t), x_{0}(x, y, t)-i y_{0}(x, y, t), 0\right) .
$$

Eq. (12) provides the complete formal solution for the quantum mechanical problem of a harmonic oscillator with variable frequency in terms of the characteristics of Eq. (8), as 
should be expected [6, 25]. Equations (12) are of the classical Hamilton type, and can be solved in general only numerically. Still, a good analytical approximation can be found under two assumptions. First, the relative change of the oscillator frequency must be small $(|1-\lambda| \ll 1)$. (This assumption holds for any reasonable experimental realization.) Second, the frequency must change either very fast $\left(\dot{\lambda} \gg \omega_{0}\right)$, or very slowly, $\left(\dot{\lambda} \ll \omega_{0}\right)$, compared to the oscillator period. It was shown [22] in a general case, that only when the frequency is changed fast in one direction and slowly in the other, an arbitrarily strong squeezing by small repeated frequency shifts can take place. Therefore our analytical approximation is good for describing the very regime we are interested in.

Let us first treat the fast limit. In this case, $\beta(t)$ can be neglected compared to $\gamma(t)$, and the equations (12) are reduced to $\dot{\alpha}=-2 i \gamma(t) \alpha^{*}(t) ; \quad \dot{\alpha}^{*}=2 i \gamma(t)^{*} \alpha(t)$. We must keep all terms in $\gamma(t)$ until we transform this system into a second-order equation for $\alpha(t)$. Now, dropping the small terms (assuming $\ddot{\lambda} \ll \dot{\lambda} \equiv v$ ), we obtain

$$
\ddot{\alpha}+v \dot{\alpha}-4 v^{2} \alpha=0 .
$$

In the case of a linear frequency change, $v=$ const, this equation is easily solved with the initial conditions $\alpha(0)=\alpha, \dot{\alpha}(0)=2 v \alpha^{*}$, yielding (see Fig. 1)

$$
\begin{aligned}
x_{0}(t, x, y) & =x_{0}(t, x)=\frac{x \sqrt{17}}{\left(2-s_{-} / v\right) e^{s_{+} t}+\left(s_{+} / v-2\right) e^{s_{-} t}} \\
y_{0}(t, x, y) & =y_{0}(t, y)=\frac{y \sqrt{17}}{\left(s_{+} / v-1\right) e^{s_{+} t}+\left(1-s_{-} / v\right) e^{s_{-}}} \\
s_{+} & =v(\sqrt{17}-1) / 2 ; \quad s_{-}=-v(\sqrt{17}+1) / 2 .
\end{aligned}
$$

In the slow regime, the terms with $\dot{\lambda}$ can be neglected. The remaining terms are of the same order, but $\gamma$-terms are very fast (oscillating with $2 \omega_{0}$ ) and average to zero over the period of the oscillator. Therefore we are left with

$$
\frac{1}{2} \dot{x}=\beta(t) y(t) ; \quad \frac{1}{2} \dot{y}=-\beta(t) x(t),
$$

or

$$
\dot{\alpha}=-2 i \beta(t) \alpha(t), \Longrightarrow \alpha(t)=\alpha(0) \exp \left[-i \frac{\omega_{0}}{2} \int_{0}^{t}\left(\lambda^{2}(s)-1\right) d s\right] .
$$

Even without solving these equations, it is clear that the slow regime cannot affect squeezing in any way: Eq. (17) describes circles in phase plane; the evolution of the Wigner function 
given by (12) therefore reduces to its (slow compared to $\omega_{0}$ ) rotation as a whole, without changing shape. This conclusion is consistent with previous work [17, 22].

The experimental realization of this proposal is rather challenging. There have been several reports of an ultrafast perturbation of optical microcavity modes using the dispersion of injected free carriers [26, 27, 28]. However, the small frequency shifts (of the order $|\lambda-1| \sim 5 \times 10^{-4}$ ) were on a picosecond timescale, which is slow with respect to the period of one optical cycle of the cavity modes. These processes are therefore in the adiabatic limit, rather than in the sudden-frequency-shift regime [17]. Thus, while they may prove useful for on-chip frequency conversion [29, 30], they are not useful for generating non-classical optical states. Moreover, there is little prospect for a repeated application of the perturbation within the sub-nanosecond lifetime of a microcavity mode.

In optical lattices occupied by cold atoms, the relatively low oscillation frequencies $(\sim 1$ $\mathrm{MHz}$ ) and precise dynamic control of the atomic potentials have allowed the demonstration of squeezed positional states [31]. The situation is also promising in the (0.1-10) GHz range, where one can use Josephson junctions as nondissipative nonlinear elements, allowing control of the circuit parameters.

If the frequency is low, so that $\hbar \omega \simeq k_{B} T$, where $T$ is $10-50 \mathrm{mK}$ (dilution refrigerator temperatures) the so-called RF SQUID [32] configuration can be used. The system consists of a high-quality superconducting tank circuit, inductively coupled to a loop containing the Josephson contact (the single junction interferometer [32]). In the dispersive mode of an RF SQUID, the effective inductance of the system becomes [32]:

$$
L_{\mathrm{eff}}=L_{T}-\frac{M^{2}}{L+\mathcal{L}(\varphi)}
$$

where $L_{T}, L$ are respectively the self-inductance of the tank circuit and of the RF SQUID loop, $M$ is their mutual inductance, and

$$
\mathcal{L}(\varphi)=\frac{L}{\beta \cos \varphi}
$$

is the Josephson inductance of the SQUID junction, which depends on the phase bias $\varphi$ across it. Here $\beta \equiv 2 \pi L I_{C} / \Phi_{0}$ and $I_{C}$ is the critical current of the SQUID Josephson junction. Therefore, by varying $\varphi$ one controls the effective inductance and eigenfrequency of the tank:

$$
\frac{\omega_{\mathrm{eff}}^{2}}{\omega_{0}^{2}}=\frac{L}{L_{\mathrm{eff}}} \approx 1+\frac{k^{2}}{(1+\beta \cos \varphi)}
$$


Here $k^{2} \equiv M^{2} / L L_{T} \ll 1$ (in practice $k \approx 0.3$ can be easily realized) is the coupling coefficient between the tank and the SQUID loop. Since for a SQUID dispersive mode $\beta<1$, the variations $\delta \omega_{0}$ of the eigenfrequency of the tank satisfy $\delta \omega_{0} \simeq(0.1-0.01) \omega_{0}$, which should be enough for our purposes.

For higher frequencies $\hbar \omega \gg k_{B} T$, a tuneable superconducting cavity could be used. In such a device, a DC SQUID is incorporated to the strip resonator [33] and $\delta \omega_{0} \simeq(0.1-$ $0.01) \omega_{0}$ as well.

For both cases, the phase-changing pulse must be sharp on the scale of $\omega_{0}$ and this is within the current experimental capabilities. The coherence time of the system is currently in the range of $1-10 \mu \mathrm{s}$, allowing for at least several cycles of frequency change, with the corresponding increase in the squeezing of the final state.

In conclusion, we have shown that the above procedure can produce squeezed states in an nonlinear superconducting oscillator, so that the fluctuations of the amplitude (phase) of the oscillator are suppressed along certain directions in phase space, which rotate with the base oscillator frequency $\omega_{0}$. By making use of this noise suppression, the measurements of the amplitude (phase) of these oscillators can reach a sensitivity below the standard quantum limit, or at least, below the thermal level.

\section{Acknowledgments}

$\mathrm{AZ}$ is grateful to B. Ivanov for valuable discussions and for pointing out references [24, 25]. FN gratefully acknowledges partial support from the National Security Agency (NSA), Laboratory Physical Science (LPS), Army Research Office (ARO), National Science Foundation (NSF) grant No. EIA-0130383, JSPS-RFBR 06-02-91200, and Core-to-Core (CTC) program supported by the Japan Society for Promotion of Science (JSPS).

[1] V.B. Braginsky and F.Ya. Khalili, Quantum Measurements (Cambridge University Press, Cambridge, 1995).

[2] J.Q. You and F. Nori, Physics Today 58, No. 11, 42 (2005).

[3] A. Zagoskin and A. Blais, Physics in Canada 63, No. 4, 215 (2007). 
[4] E. Il'ichev et al., Phys. Rev. Lett. 91, 097906 (2003); A. Izmalkov et al., Phys. Rev. Lett. 93, 037003 (2004); I. Chiorescu et al., Nature 431, 159, (2004); M. Grajcar et al., Phys. Rev. Lett. 96, 047006 (2006); S.H.W. van der Ploeg et al., Phys. Rev. Lett. 98, 057004 (2007).

[5] E. Il'ichev et al., Low Temp. Phys. 30, 620 (2004).

[6] V.P. Bykov, Sov. Physics - Uspekhi 34, 910 (1991).

[7] M. Orszag, Quantum Optics (Springer, New York, 1999).

[8] C.W. Gardiner and P. Zoller, Quantum Noise (Springer, Berlin, 2004).

[9] M. Artoni and J.L. Birman, Phys. Rev. B 44, 3736 (1991).

[10] X. Hu and F. Nori, Phys. Rev. B 53, 2419 (1996).

[11] X. Hu and F. Nori, Physica B 263, 16 (1999).

[12] X. Hu and F. Nori, Phys. Rev. Lett. 76, 2294 (1996); Phys. Rev. Lett. 79, 4605 (1997).

[13] X. Hu and F. Nori, Squeezed Quantum States in Josephson Junctions, UMpreprint (1996); X. Hu, Univ. of Michigan (UM) Thesis (1996); see also http://www-personal.umich.edu/ nori/squeezed.html.

[14] J. Janszky and An. V. Vinogradov, Phys. Rev. Lett. 64, 2771 (1990).

[15] B. Yurke et al., Phys. Rev. Lett. 60, 764 (1988); B. Yurke et al., Phys. Rev. A 39, 2519 (1989).

[16] D. Rugar and P. Grutter, Phys. Rev. Lett. 67, 699 (1991).

[17] R. Graham, J. Mod. Opt. 34, 873 (1987).

[18] G.S. Agarwal and S.A. Kumar, Phys. Rev. Lett. 67, 3665 (1991).

[19] J. Janszky and P. Adam, Phys. Rev. A 46, 6091 (1992).

[20] M.S. Abdalla and P.K. Colegrave, Phys. Rev. A 48, 1526 (1993).

[21] T. Kiss, J. Janszky, and P. Adam, Phys. Rev. A 49, 4935 (1994).

[22] I. Averbukh, B. Sherman, and G. Kurizki, Phys. Rev. A 50, 5301 (1994).

[23] M. J. Everitt et al., Phys. Rev. A 69, 043804 (2004).

[24] A. S. Abyzov and B. A. Ivanov, Zh. Eksp. Teor. Fiz. 76, 1700 (1979).

[25] A. Perelomov, Generalized coherent states and their applications (Springer, Berlin, 1986).

[26] I. Fushman et al., Appl. Phys. Lett. 90, 091118 (2007).

[27] S. F. Preble, Q. Xu, and M. Lipson, Nature Photonics 1, 293 (2007).

[28] M.W. McCutcheon, A. G. Pattantyus-Abraham, G. W. Rieger, and J. F. Young, Optics Express 1, 293 (2007).

[29] M. Notomi, H. Taniyama, S. Mitsugi, and E. Kuramochi, Phys. Rev. Lett. 97, 023903 (2006). 
[30] M. Notomi and S. Mitsugi, Phys. Rev. A 73, 051803(R) (2006).

[31] P. Rudy, R. Ejnisman, and N. P. Bigelow, Phys. Rev. Lett. 78, 4906 (1997).

[32] A. Barone and G. Paterno, Physics and Applications of the Josephson Effect (Wiley, New York, 1982).

[33] M. Sandberg et al., cond-mat 0801.2478v1. 

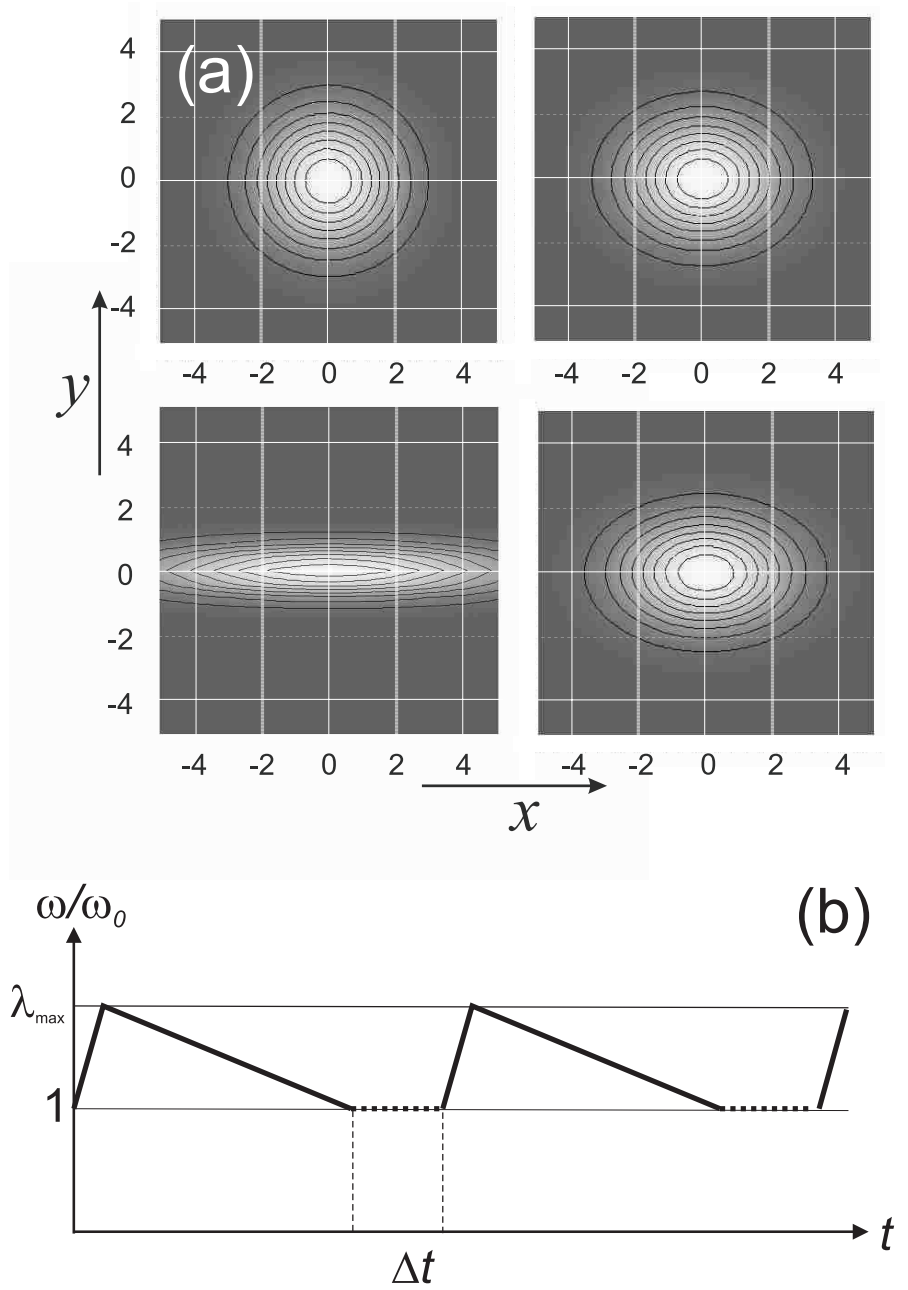

FIG. 1: Squeezing of a thermal state, with $k_{B} T=4 \omega_{0}$, by repeated frequency shifts, with $\lambda_{\max }-1=$ 0.05. (a) Contour plots of the approximate expression of the Wigner function in the interaction representation (see Eqs. $(12,15,17)$ ) are shown as a function of the quadrature variables, $x$ and $y$. Clockwise starting from the upper left: initial thermal state, the state after one, two, and ten cycles. The dark background corresponds to $W=0$, and white to $W=0.08$. (b) A schematic frequency-versus-time dependence necessary to produce the results of Fig. 1a. The idle periods $\Delta t$ are chosen to ensure that every fast shift occurs in the same phase with respect to the quadrature coordinates. Here one must account for the fact that in the Schroedinger representation the whole phase plane rotates around the origin with the base oscillator frequency $\omega_{0}$. An additional rotation of the Wigner function as a whole during the slow shift, Eq. (18), can be neglected in comparison, since the additional phase $|\delta \theta| \leq 0.5 \omega_{0} t\left|\lambda_{\max }^{2}-1\right| \ll \omega_{0} t$. 